\title{
Nikolaus Konietzko zum 70. Geburtstag
}

\section{Nikolaus Konietzko on the Occasion of his $70^{\text {th }}$ Birthday}

Autor

Institut

\section{H. Teschler}

Ruhrlandklinik, Abteilung Pneumologie-Universitätsklinik
Bibliografie

Dol $10.1055 / \mathrm{s}-0028-1103475$

Pneumologie 2009; 63: 56-57

(c) Georg Thieme Verlag KG

Stuttgart · New York

ISSN 0934-8387

Korrespondenzadresse

Prof. Dr. med. Helmut Teschler Ruhrlandklinik

Abteilung Pneumologie-

Universitätsklinik

Tüschener Weg 40

45239 Essen

teschlerh@t-online.de
Am 6. Dezember 2008 feiert Nikolaus Konietzko seinen 70. Geburtstag. Zwar ist dieser Tag nur eine kurze Episode in einem langen, erfolgreichen Arzt- und Pneumologenleben, doch runde Geburtstage wie diese lassen Ereignisse und Entwicklungen der Vergangenheit ins Gedächtnis rufen, die für die künftigen Weichenstellungen von Bedeutung sind.

Zur Welt gekommen ist unser Jubilar am Nikolaustag des Jahres 1938 als viertes von sechs Kindern in Kieferstädtel im Kreis Gleiwitz in Oberschlesien. Die ersten Lebensjahre verbrachte er in seiner Geburtsstadt. Die Wirren des Jahres 1945 führten zu Flucht und Vertreibung aus der Heimat, die seitdem zu Polen gehört. Die Familie wurde schließlich in Neumarkt in der Oberpfalz sesshaft.

Nach dem Abitur am humanistischen Gymnasium im Jahre 1958 studierte er Humanmedizin an der Ludwig-Maximilians-Universität in München. Seine auch heute noch große Vorliebe für reichlich „gute Butter“ leitet er - vielen Gurus der Ernährungswissenschaften zum Trotz - aus den Ergebnissen seiner Promotionsarbeit mit dem Thema „Der Einfluss ungesättigter Dicarbonsäuren auf den Fettstoffwechsel der Leber“ $\mathrm{ab}$.

Im Jahre 1963 erhielt er die Approbation. Die umfangreiche berufliche Biografie von Professor Nikolaus Konietzko soll hier nicht in voller Länge wiederholt werden. In der gleichen Zeitschrift kann man diese an anderer Stelle (Pneumologie 2003; 57: 771 - 773) detailliert nachlesen. Nur so viel: Seine internistisch-pneumologische Ausbildung startete er an der Medizinischen Universitätsklinik in Gießen bei Professor Thure von Uexkuell, er verbrachte ein Jahr als Fellow bei Professor Robert Carton an der University of Illinois in Chicago, studierte an dieser Universität bei Professor Ruy Lourenco die Anwendung von Radio-Isotopen zur Bestimmung der mukoziliaren Clearance und wechselte danach an die neu gegründete Universität zu Ulm. In der Sektion Pneumologie, die von Professor Heinrich Matthys geleitet wurde, habilitierte er mit dem Thema „Lungenfunktionsprüfung mit Radionukliden“. Danach begann eine neue Schaffensperiode. Professor Werner Maassen holte ihn 1976 nach Essen und übertrug ihm die Leitung der neu gegründeten Abteilung für „Innere Medizin und Funktionsdiagnostik“ der Ruhrlandklinik. Im Jahre 1982 verlieh ihm die Universität Essen den Titel „außerplanmäßiger Professor“. Nach dem Ausscheiden von Professor Maassen wurde er Ärztlicher Direktor der Klinik und Chefarzt der Abteilung Pneumologie.

Unter seiner Leitung entwickelte sich die Ruhrlandklinik in den Folgejahren zu einem überregionalen Zentrum für Pneumologie und Thoraxchirurgie. Die Weiterentwicklung zu einer der leistungsstärksten Einrichtungen dieser Art in Europa verdankt die Klinik strukturellen und personellen Entscheidungen, die Nikolaus Konietzko zur rechten Zeit und mit großem Weitblick getroffen hat. Dazu zählt auch die Realisierung eines Kooperationsvertrages mit dem Universitätsklinikum Essen.

Er erkannte frühzeitig die Notwendigkeit der hohen Spezialisierung einer Lungenfachklinik moderner Prägung, wozu er auch die kontinuierliche Weiterentwicklung von Pneumologie und Thoraxchirurgie auf Augenhöhe zählte. Im Jahre 1988 holte er Professor Ulrich Costabel an seine Klinik und übertrug ihm den Aufbau einer Abteilung mit Schwerpunkt „Interstitielle Lungenerkrankungen", die heute weltweites Ansehen genießt. Ihm verdankt die Klinik außerdem die Einrichtung einer Spezialabteilung für Patienten mit Mukoviszidose im Erwachsenenalter, die Eröffnung eines leistungsstarken Schlaflabors mit Fokussierung auf die respiratorische Schlafmedizin und zudem - wenn auch in bescheidenem Umfang - den Einstieg in die Respiratorentwöhnung von langzeitbeatmeten Patienten mit 


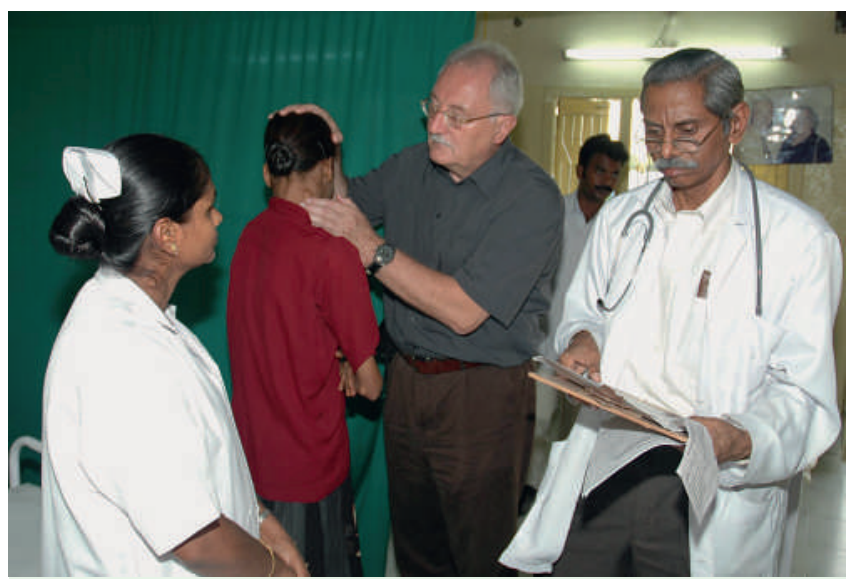

Abb. 1 Arzt aus Leidenschaft (Indisches Tuberkulosekrankenhaus, Projekt Deichmann-Stiftung).

COPD und anderen chronischen Lungenerkrankungen. Daneben war er Leiter der europaweit größten Studie zur Behandlung des Lungenemphysems auf dem Boden eines schweren Alpha 1-Proteinaseinhibitormangels durch Substitutionstherapie.

Eine weitere Entscheidung erwies sich ebenfalls als weitsichtig und erfolgreich. Bereits wenige Jahre nach der ersten erfolgreichen unilateralen Lungentransplantation durch Joel Cooper in Toronto erkannte er deren enormes Potenzial für Patienten mit terminalen Lungenerkrankungen. Folgerichtig baute er eine Kooperation mit dem Transplantationszentrum des Universitätsklinikums Essen auf. Er ermöglichte dem damaligen Oberarzt und heutigen Chefarzt der Abteilung Thoraxchirurgie der Ruhrlandklinik, Herrn Professor Stamatis, die Hospitation in Toronto und trug so dazu bei, dass 1991 die erste Lungentransplantation am Standort Essen durchgeführt werden konnte.

Heute kann Professor Konietzko auf die Früchte eines arbeitsund erfolgreichen Berufslebens zurückblicken: die Ruhrlandklinik geht den von ihm eingeschlagenen Weg der universitären Ausrichtung ihres Leistungsspektrums konsequent weiter. Am 1. April 2009 wird ein Trägerwechsel vollzogen. Die Klinik wird dann den Namen „Ruhrlandklinik, Westdeutsches Lungenzentrum am Universitätsklinikum Essen“ tragen. Auf diesem nicht immer einfachen Weg war er uns auch nach seinem Ausscheiden aus der Klinik mit all seiner Erfahrung und seinem Weitblick ein verlässlicher und wertvoller Berater.

Seine Mitarbeiter schätzen Nikolaus Konietzko als einen außergewöhnlichen Menschen, einen Humanisten reich an Lebenserfahrung und ausgestattet mit immenser klinischer Erfahrung. Er verabschiedete sich aus dem aktiven Dienst mit der Feststellung, dass der Arztberuf für ihn stets ein Traumberuf war und nach wie vor ist. Wir, die wir mit ihm die Zukunft der Klinik maßgeblich gestalten durften, haben in ihm einen Arzt von beispielgebendem Verantwortungsgefühl sowie mit unermüdlichem Arbeitseifer und ausgeprägtem Pflichtbewusstsein erleben dürfen, der als akademischer Lehrer bei seinen Schülern und Studenten außerordentlich beliebt war. Gleichzeitig freuen wir uns, dass seine physische und psychische Fitness es ihm erlaubt, den Traum vom Arztberuf in seinem Engagement für AIDS- und Tuberkulosekranke im Rahmen eines Tuberkulosekrankenhauses in Indien, einem Projekt der Deichmann-Stiftung (Wort und Tat) ( Abb. 1), auch jetzt noch in die Tat umzusetzen.

Die Weiterentwicklung der Deutschen Gesellschaft für Pneumologie hat Professor Nikolaus Konietzko in seiner aktiven Zeit maßgeblich geprägt. Mit seinem Namen sind die Restrukturierung unserer Fachgesellschaft ab 1990, die Etablierung von wissenschaftlichen Sektionen, die Einrichtung von Arbeitsgruppen zur Erarbeitung von Empfehlungen (später Leitlinien) und - in Kooperation mit Professor Fabel - die Herausgabe des Weißbuches Lunge verbunden. Im Jahre 1995 übernahm er das Amt des Präsidenten und letztmals in der jüngeren Geschichte der DGP in Personalunion des Tagungspräsidenten unserer Gesellschaft. Er stärkte das Gewicht der deutschen Pneumologie in den europäischen Fachgesellschaften wie der Societas Europaean Pneumologica (SEP) und der später gegründeten European Respiratory Society (ERS), für die er 1991 als Chairman den Kongress in Berlin ausrichtete. Außerdem war er lange Jahre Geschäftsführer der Rheinisch-Westfälischen Gesellschaft für Lungen- und Bronchialheilkunde.

Mit der Verleihung der Ehrenmitgliedschaft haben die Deutsche Gesellschaft für Pneumologie und Beatmungsmedizin und die Rheinisch-Westfälische Gesellschaft für Lungen- und Bronchialheilkunde dem Jubilar ihre Wertschätzung für sein berufliches und wissenschaftliches Lebenswerk gebührlich bekundet.

Als Präsident der Deutschen Gesellschaft für Pneumologie und Beatmungsmedizin und als sein Nachfolger als Chefarzt der Abteilung Pneumologie-Universitätsklinik der Ruhrlandklinik bedanke ich mich bei ihm für sein großes Engagement zum Wohle der deutschen Pneumologie und wünsche dem Geburtstagskind noch viele lange und erfüllte Jahre im Kreise seiner Familie und seiner zahlreichen Freunde.

\section{Ad multos annos, lieber Nikolaus Konietzko!}

Prof. Dr. med. Helmut Teschler

Als Chefärzte der Ruhrlandklinik, die eine lange Strecke ihres beruflichen Weges Seite an Seite mit Nikolaus Konietzko gehen durften, möchten wir uns dieser Laudatio, dem Dank und den Glückwünschen anschließen. Wir danken einem großen akademischen Lehrer für die Weitergabe eines profunden, heute selten gewordenen klinischen Wissens und einem kollegialen Klinikdirektor für die wertvolle Unterstützung beim Auf- und Ausbau unserer Abteilungen. Wir wünschen dem Jubilar noch viele gesunde und glückliche Jahre mit unveränderter Freude an den schönen Dingen des Lebens; wir wollen noch lange von seiner Lebenserfahrung und Altersweisheit bereichert werden!

Prof. Dr. med. Ulrich Costabel

Prof. Dr. med. Georgios Stamatis 